[Original]

\title{
Die unendliche Geschichte von Michael Ende-
}

- ein Märchenroman-eine Phantasieerzählung - ein sozialpolitisches Essay

\author{
Mieko NAKAO \\ The Division of German Language, \\ School of Nursing and Medical Technology, University of Occupational and Environmental Health, Japan. \\ Yahata nishi-ku, Kitakyushu 807, Japan
}

Abstract: The novel "The Never Ending Story" by Micheal Ende was No. 1 on the weekly bestseller-list of the German news magazine 'Der Spiegel' for at least 3 years and 3 months. This long-term run was an outstanding record for any book published in post-war Germany. The 'Reader', a boy called Bastian, enters the story himself and in this world of Fantasy he undergoes many experiences, struggles with different thoughts, and gradually matures after several defeats, errors, disappointments and victories, becoming more and more conscious of his true "self". The literary style of this book full to the brim with its fantastic words is aimed first at the young generation, but at the same time, it also delivers a strong message to all adults of the 20th century who seem to have lost their sense for Fantasy - encouraging them with an energetic appeal to re-discover Fantasy and poetics in order to create a balance with the too realistic-minded world of adults. Being structured in an ambigious style and combined with numerous images, this novel suggests a possible way to correct the difficulties in our society. Therefore, this novel aims not only at the young generation but also carries a strong message for all adults.

Key words: Fantasy, dream, reality, self-recognition, interrelation between book and reader.

(Received 15 February 1995, accepted 26 May 1995)

\section{Einleitung}

Schon mit seinem Märchenroman "Momo" gelang Micheal Ende der Sprung in die Bestsellerliste. Die Thematik seiner Romane spricht nicht nur inhaltlich die Leser an, sondern auch die Sprache, in der sie verfaßt sind, erleichtert durch ihre Einfachheit und Ungeziertheit stilistischer Raffinessen die Lektüre für Jung und Alt. Endes Beschreibugen von Naturbildern, Ereignissen und den darin vorkommenden Fabelwesen sind wie Einladungen an 
den Leser, sich aus den Hinweisen selber ein konkretes Bild zu machen. Diese Freiheit für den Leser mag auch ein wichtiger Schlüssel sein für den weltweiten Erfolg seiner Bücher, da Menschen von verschiedensten Kulturen sich ihre "Story" vervollständigen können. Damit hatte M. Ende ein sicheres Erfolgsrezept gefunden für seinen größten Bestseller, die Unendliche Geschichte, die noch märchenhafter Jugendliche und Erwachsene anspricht. Das Buch ist nicht nur in deutschsprachigen Ländern ein großer Erfolg, sondern in 24 Fremdsprachen übersetzt wurde daraus ein weltbekanntes Buch. Für dieses Werk wurde der Autor mit mehreren Auszeichnungen geehrt, von denen der deutsche Jugendbuchpreis, der europäische Jugendbuchpreis und der große Preis der deutschen Akademie für Kinder- und Jugendbücher sicher die wichtigsten sein dürften. Kein Wunder also, wenn sich die Filmindustrie schon sehr bald für diesen Roman interessierte. Der Regiseur Wolfgang Petersen, der mit dem Film 'Das Boot' Weltruhm errang, wagte sich an das schwierige Unternehmen, das viele Freunde des Buches für unmöglich hielten. Mit einem Kostenaufwand von 60 Millionen Mark entstand so einer der teuersten deutschen Filme der Nachkriegszeit. Der Autor ist allerdings über das Ergebnis des Filmes nicht sehr erfreut, da dieser doch sehr vom Buch abweicht und daher der eigentliche Sinn der Erzählung nicht mehr erkennbar werde.

Die Unendliche Geschichte ist in der immer reichhaltiger werdenden Phantasie-Literatur des 20. Jahrhunderts sicher kein Neuling. Auch lassen sich einige formale und inhaltliche Paralellen $\mathrm{zu}$ anderen Erfolgserzählungen finden, wie z. B. zum Kleinen Prinzen von Exuperie, wo es auch um die Diskrepanz zwischen Kinder- und Erwachsenenwelt, echter Verantwortung und Liebe geht. Manche Massenmedien urteilen sehr kritisch über das Werk, wie z. B. das Magazin 'Der Stern' [1]: "Viele, die sich vor Computer-Staat und Umweltzerstörung fürchten, flüchten in Märchen für Erwachsene. In der Fantasiewelt kämpfen Fabelwesen gegen die Mächte der Finsternis - und immer siegt das Gute über das Bösse." -So grob verallgemeinert sind dies sicher keine Argumente, diesem Märchen den literarischen Charackter absprechen zu können, in dem man es zum einfachen Erfolgsschlager abstempelt. Schon in sehr frühen literarischen Zeiten können in fast allen Sprachen der Welt Märchen, Heldenepen und Phantasiegeschichten nachgewiesen werden. Die psycho-analytische Schule G. G. Jungs z. B. versteht die Märchenbilder als Manifestationen überindividuellen, zeitlosen seelischen Geschehens. So gesehen läßt sich das Werk von M. Ende als eines der erfolgreichsten Gegenwartsmärchen durchaus organisch in die lange Geschichte der Märchenliteratur einreihen. 


\section{Zum Inhalt}

Zuerst begegnen wir dem Helden der Geschichte, dem 10-jährigen Jungen namens Bastian Bux, in der Gestalt des Antihelden, welcher Probleme hat zu Hause und in der Schule und der im Bücherlesen Vergessen sucht. Sicherlich ist daher der Anklang des Namens Bux an das englische 'books' nicht zufällig. Bastian schwänzt den Unterricht und versteckt sich auf dem Dachboden der ungeliebten Schule, um das aus einem Antiquariat entfuhrte Buch, dessen Titel ihn so begeisterte, zu Gemüte zu führen. So sitzt Bastian auf dem Dachboden seiner Schule auf einer alten staubigen Turnmatte zwischen allerlei Gerümpel und einem ausgedienten Skelett - sicherlich kein idealer Platz zum Träumen. Aber gerade dort wird seine Traumreise nach Phantasien beginnen. Unbekümmert um den Schulbetrieb, die Klingelzeichen, den entfernten Lärm der Schulfreunde vertieft er sich in die Erzählung. Er liest von einem Land Phantasien, das in großer Gefahr ist, vernichtet zu werden. Die friedlichheitere Gegenwelt zu unserer furchtbar realen Welt ist vom Untergang bedroht. Ein unheimliches, unfaßbares 'Nichts' zerfrißt Wälder und Blumenfelder, löst Land und Meer buchstäblich in Nichts auf und dringt unaufhaltsam vor. Der Jägerjunge Atreju (=andre you) wird im Namen der todkranken "Kindlichen Kaiserin" beauftragt, nach Rettung vor dem Nichts zu forschen. Atreju reitet alleine los auf eine Expedition voller Prüfungen, Gefahren aber auch guter Belehrungen. Bastian Bux liest von Atrejus Abenteuern so heißhungrig, wie andere Kinder von dem gefesselt sind, was z. B. Robinson in der Einöde oder was dem Jungen Jim auf der Schatzinsel widerfuhr. Doch da passiert etwas Besonderes, womit M. Ende seinen Lesern sicher auch etwas mitteilen will: Lesen heißt selbst Teil der Geschichte zu werden, oder anders gesagt: durch die eigene Phantasie soll eine Geschichte zum Leben erweckt werden. In der unendlichen Geschichte wird auf einmal der Leser Bastian angesprochen und zwar als möglicher Retter Phantasiens. Die Kindliche Kaiserin - krank wie sie ist - wendet sich selbst direkt an Bastian und bittet ihn, einzusteigen in die Geschichte und mitzumachen. Nur er könne das unheimliche Nichts aufhalten, denn Phantasien gehe zugrunde, weil keine Menschen mehr in das Sagenreich kommen. Die Menschen glauben nicht mehr an Phantasien, obwohl es aus den Träumen und Hoffnungen der Menschheit besteht und nur von ihnen lebt.

Hierin liegt der Schlüssel der "Unendlichen Geschichte", das ist die Botschaft, die M. Ende dem Leser geben möchte: Ohne Phantasien wird die Menschenwelt öd und leer. Ohne diese Gegenwelt hätten wir der Realität nichts entgegenzusetzen. "Menschen, die ihre Hoffnung und Träume 
verloren haben, sind leicht zu beherrschen" [2], knurrt der Werwolf 'Gmork', der die Interessen der Mächtigen der Erde vertritt, denen die Vernichtung Phantasiens gar nicht schnell genug voranschreiten kann. Der Autor beklagt, daß für viele von uns diese Gegenwelt schon tot sei, dieses Phantasien, diese Sagenwelt, dieser Kinderglaube, aus dem man herausgewachsen zu sein glaubt. Man nehme nur noch wichtig, was wirklich existiere. Vom Reich der Phantasie bleiben dann nur noch verkümmerete oder mißbrauchte Bruchstücke: die Vergnügungen der Unterhaltungsindustrie, die Konsumträume.

Der Bücherwurm Bastian neigte bisher selber zu der Ansicht, Bücher seien "einfach nur Geschichten", spannend oder langweilig, die er zur Ablenkung und Flucht vor sich und seiner Realität mißbraucht. Dann aber wird er von diesem unheimlichen Buch in die Zange genommen und auf die Probe gestellt: Glaubt er an die Not Phantasiens, oder ist das für ihn auch wieder "bloß eine Geschichte"? Glaubt er daran, daß die Kindliche Kaiserin ausgerechnet ihn für den einzigen Erdbewohner hält, der sie retten und ihr Reich neu erschaffen kann?

Nach nagender Ungewißheit und quälenden Zweifeln wagt er den Dialog und schließlich auch den Sprung in die Geschichte: Er glaubt an die Kindliche Kaiserin, er will zu ihr, er ist bereit, sie und den furchtlosen Atreju zu retten. Mit dem Eintauchen Bastians in die Weiterentwicklung der Geschichte wird er selbst nun zur Hauptfigur. Alles, was er sich bisher so vergebens gewünscht hatte, gelingt ihm: stattlich auszusehen, kräftig und mutig zu sein, harte Proben bestehen zu können, dank des 'Auryn', dem beschützenden Symbol der unumschränkten Herrschergewalt in Phantasien, das er von der Kaiserin bekommen hatte. Er gefällt sich als der gefeierte Held und gerät gerade deshalb nun in seine eigene Krise - er ist nun von Vernichtung bedroht. Langsam verliert er sein Gedächtnis an seine eigene reale Welt, in die er nicht mehr zurückkehren möchte. Sein Stolz läßt ihn Fehler begehen, hartherzig werden und droht sogar die tiefe Freundschaft zu Atreju zu zerstören. Er muß, um der Vernichtung aus dem Weg zu gehen, die magische Kraft des Auryn ablegen, um in seine eigene Welt wieder zurückkehren $\mathrm{zu}$ können. Dank der Hilfe von Atreju gelingt ihm dies im letzten noch möglichen Augenblick. Gereift und verändert wacht Bastian auf dem Speicher auf: Er ist jetzt nicht mehr der ängstliche, schwächliche Junge, er kann sich annehmen, wie er ist, er hat gelernt, seine Realität voll zu akzeptieren. Etwas bange zwar, aber doch voll Entschlossenheit verläßt er die Schule und tritt den Heimweg an. Auch hier hat sich Vieles verändert: Der bisher kühle Vater schließt ihn herzlich in die Arme. Der anfangs sehr spröde Herr Koreander, der Antiquariat, dem er das Buch weggenommen hatte, und das nach dem Aufwachen spurlos verschwunden war, ist genauso verändert. Geduldig hört 
er sich die Geschichte Bastians an, und da auch er zu den Pilgern nach Phantasien gehört, gibt er Bastian seine Erfahrung weiter mit den Worten: "Es gibt Menschen, die können nicht nach Phantasien kommen - und es gibt Menschen, die können es, aber sie bleiben für immer dort. Und dann gibt es noch einige, die gehen nach Phantasien und kehren wieder zurück. So wie du. Und die machen beide Welten gesund." (S. 426)

Wie der Mensch Geschichten braucht, braucht die Geschichte den Menschenoder: Was bedeutet richtiges Lesen?

In der Geschichte stürzt Bastian rein zufällig in den Antiquariatenladen, da er wieder einmal vor den ihn verspottenden Klassenkameraden auf der Flucht ist. Dort begegnet er dem schrulligen Ladenbesitzer, der wie Bastian gleich drei mit den gleichen Buchstaben beginnende Namen hat: Karl Konrad Koreander - ein Hinweis auf die Ähnlichkeit der beiden, die aber erst am Schluß des Buches klar wird. Noch trennen die beiden Welten, denn Herr K. K. Koreander empfängt den Jungen nicht gerade höflich: "Hör zu, mein Junge, ich kann keine Kinder leiden. Für mich sind Kinder nichts als Schreihälse,.. die die Bücher mit Marmelade vollschmieren und die Seiten zerreißen....Außerdem gibt es bei mir keine Bücher für Kinder, und andere verkaufe ich dir nicht (S. 6-7)." Für den Büchernarr Bastian ändert sich diese rauhe Umgebung, als er ein Buch mit dem Titel "Die Unendliche Geschichte" findet. Endlich hatte er etwas gefunden, von dem er schon immer geträumt hatte: "Wer niemals ganze Nachmittage lang mit glühenden Ohren und verstrubbeltem Haar über einem Buch saß und las und las und die Welt um sich herum vergaß, nicht merkte, daß er hungrig war oder fror....Wer niemals heimlich beim Schein einer Tachenlampe unter der Bettdecke gelesen hat, weil Vater oder Mutter oder sonst irgendeine besorgte Person einem das Licht ausknipste mit der gut gemeinten Begründung, man müsse jetzt schlafen, da man doch morgen früh aus den Federn sollte.... Wer niemals offen oder im geheimen bittere Tränen vergossen hat, weil eine wunderbare Geschichte zu Ende ging und man Abschied nehmen mußte von den Gestalten, mit denen man gemeinsam so viele Abenteuer erlebt hatte, die man liebte und bewunderte, um die man gebangt und für die man gehofft hatte, und ohne deren Gesellschaft einem das Leben leer und sinnlos schien.... Wer nichts von alledem aus eigener Erfahrung kennt, nun, der wird wahrscheinlich nicht begreifen können, was Bastian jetzt tat." Er stiehlt das Buch, weil es für ihn “das Buch der Bücher" (S. 11) ist. Endlich "eine Geschichte, die niemals zu Ende geht".

Zuerst ist Bastian ein gewöhnlicher Büchernarr, der in die Geschichten flieht vor seiner eigenen Wirklichkeit, wodurch er aber immer mehr von dieser entfernt wird. Seine Schulkameraden verspotten ihn daher als Schwindler. 
und Träumer. Dies bestätigt er durch seine Reflexion, als er mit dem Lesen begonnen hatte, und ihn die Turmuhr mit ihrem Glockenschlag plötzlich wieder in seine Wirklichkeit zurückrief: "Er war froh, daß die Unendliche Geschichte nichts mit ihr (= seiner Wirklichkeit) zu tun hatte." (S. 25) Er mochte keine Bücher, in denen ihm auf eine schlechtgelaunte und miesepeterige Art die ganz alltäglichen Begebenheiten aus dem ganz alltäglichen Leben irgendwelcher ganz alltäglicher Leute erzählt wurden.

Davon hatte er ja schon in Wirklichkeit genug, wozu sollte er auch noch davon lesen? Und in dieser Art von Büchern sollte man immer, mehr oder weniger deutlich, zu was gekriegt werden." (S. 26) Mit diesen Sätzen Bastians spricht Ende seine Kritik an der modernen deutschen Literatur aus, die besonders seit der Nachkriegszeit immer nüchterner wurde und mit sozialpolitischer Kritik analysierte und den Lesern entsprechende Denkanstöße vermittelte. Dieses sehr praktisch ausgerichtete "Realitätsdenken" entspricht ganz und gar nicht dem Denken Bastians - und das heißt hier sicher dem Denken des Autors, der in seiner Rede auf dem 20. Weltkongress für Kinderund Jugendbücher in Tokyo seine literarische Intention folgendermaßen formulierte: "Bei meiner Arbeit bin ich nicht von pädagogischen oder didaktischen Prinzipien geleitet....Die sichtbare Form meiner Bücher hat nur poetische und künstlerische Gründe." [3]

Bastians Interesse gilt Büchern, "die spannend waren oder lustig oder bei denen man träumen konnte, Bücher, in denen erfundene Gestalten fabelhafte Abenteuer erlebten und wo man sich alles mögliche ausmalen konnte."(S. 26) Und das konnte Bastian - ja, es war wahrscheinlich das einzige, was er wirklich konnte: "Sich etwas vorstellen, so deutlich, daß er es fast sah und hörte. Wenn er sich selbst seine Geschichten erzählte, dann vergaß er manchmal alles um sich herum und wachte erst am Schluß auf wie aus einem Traum." (S. 26)

Diese Fähigkeit läßt den Bastian dann später selbst in die Geschichte einsteigen, aber dann muß er dazulernen: Er darf die Welten nicht trennen, sondern erst in ihrer Verbindung können beide füreinander fruchtbar werden. Etwas von diesem Geheimnis scheint er zu ahnen, als er zu Beginn der Lektüre folgende Gedanken äußert: "Ich möchte wissen, was eigentlich in einem Buch los ist, solang es zu ist. Natülich sind nur Buchstaben drin, die auf Papier gedruckt sind, aber trotzdem - irgend etwas muß doch los sein, denn wenn ich es aufschlage, dann ist ja da auf einmal eine ganze Geschichte. Da sind Personen, die ich noch nicht kenne, und es gibt alle möglichen Abenteuer und Taten und Kämpfe - und manchmal ereignen sich Meeresstürme, oder man kommt in fremde Länder und Städte. Das ist alles doch drin im Buch. Man muß es lesen, damit man's erlebt, das ist klar. Aber drin ist es schon vorher. Ich möcht' wissen, wie?" (S. 16) 
Er kommt diesem Geheimnis etwas näher, als er im Land Phantasien auf Leute trifft, die nichts sprechen, sondern ein Würfelspiel machen. Sein Begleiter Argax erklärt es ihm: "Sie können nichts mehr erzählen. Sie haben die Sprache verloren. Darum habe ich dieses Spiel für sie ausgedacht. Es beschäftigt sie, wie du siehst. Und es ist sehr einfach. Wenn du einmal nachdenkst, dann mußt du zugeben, daß alle Geschichten der Welt im Grunde nur aus sechundzwanzig Buchstaben bestehen. Die Buchstaben sind immer die gleichen, bloß ihre Zusammensetzung wechselt. Aus den Buchstaben werden Wörter gebildet, aus den Wörtern Sätze, aus den Sätzen Kapitel und aus den Kapiteln Geschichten... Wenn man es sehr lang spielt, jahrelang, dann ergeben sich manchmal durch Zufall Wörter... Wenn man es aber hundert Jahre, tausend Jahre, hunderttausend Jahre immer weiterspielt, dann muß nach aller Wahrscheinlichkeit dabei durch Zufall auch einmal ein Gedicht herauskommen. Und wenn man es ewig spielt, dann müseen dabei alle Gedichte, alle Geschichten der Geschichten und sogar diese Geschichte, in der wir beide uns gerade unterhalten. Das ist logisch, nicht wahr?"-"Das ist entsetzlich", sagte Bastian (S. 367).

Er, der sich auf das Abenteuer eingelassen hatte, selber den Verlauf der Geschichte in die Hand zu nehmen, er, der es gewagt hat, seine Umwelt aus seinen Wünschen erstehen zu lassen, er weiß, daß nicht der Zufall Gedichte schreiben kann, und daß aus diesem Spiel nie eine Geschichte erstehen wird. Er lebt ganz in der Phanatsie, ist ganz in ihre Exstase eingefangen, aber gerade deshalb ist er nun auf der Suche nach dem Weg zurück, denn das Symbol auf dem Buch besagt ja, daß sich die Schlange der Realität und der Phantasie gegenseitig in den Schwanz beißen und keine die andere loslassen darf: nur die Verbindung von beiden kann die Menschen weiterbringen. Dieses Zeichen des Auryns ist unter der Bezeichnung "Uroboros' bekannt als uraltes Symbolbild der ewigen Wiederkehr. Laut Knaurs Lexikon der Symbole "stellt (das Symbol) eine aussagekräftige Metapher einer zyklischen Wiederholung dar - etwa des Kreislaufes der Zeiten, der Weltuntergänge und Neuschöpfungen, des Sterbens und der Neugeburt, im abgeleiteten Sinn auch der Ewigkeit" [4], und so untermalt das Symbol auf dem Auryn die Bedeutung der 'unendlichen' Geschichte. Aber bevor Bastian zurückkehren kann, muß er die Wirklichkeit, wie er sie bisher gesehen und erlebt hat, vergessen: er wird wieder zum kleinen Kind - er muß zurückfinden zu einer naiven Verbindung zwischen Wunsch, Traum, Hoffnung und Wirklichkeit. Erst als er über den narzistischen Wunsch, geliebt zu werden, zu seiner Aufgabe findet, selber lieben zu wollen, kann er sich selbst annehmen wie er ist. Er weiß, daß er seinem Vater das 'Wasser des Lebens' bringen muß, damit auch er über den Verlust der Mutter hinwegkommt und wieder ein froher, hoffnungsvoller Vater werden 
kann. Gereift durch diese Erfahrung kehrt Bastian aus dem Traum auf dem Dachboden zurück und er muß erstaunt feststellen, daß das Buch nicht mehr da ist. Er hat das Buch verinnerlicht, so daß es aufgehört hat, eine eigene Existenz zu sein, die man wieder ins Bücherregal zurücksteckt, als ob nichts geschehen wäre. Als er dem Buchhändler den Diebstahl und den Verlust des Buches beichten will, öfnet auch dieser in unerwarteter Freundlichkeit sein Herz: "Jede wirkliche Geschichte ist eine unendliche Geschichte", eröfnet ihm Herr Koreander und meint: "Es gibt eine Menge Türen nach Phantasien, mein Junge. Es gibt noch mehr solche Zauberbücher. Viele Leute merken nichts davon. Es kommt eben darauf an, wer ein solches Buch in die Hand bekommt." (S. 427) Bastian fragt nach: "Dann ist die unendliche Geschichte für jeden anders?" Herr Koreander: "Das will ich meinen,.. außerdem gibt es nicht nur Bücher, es gibt auch noch andere Möglichkeiten nach Phantasien und wieder zurück zu kommen. Das wirst du noch merken." (S. 427)

Auch der Leser des Buches wird nicht weniger als an 16 Stellen in der Geschichte mit einer angefangenen Episode allein gelassen und mit folgenden Worten vertröstet: "Aber das ist eine andere Geschichte und soll ein andermal erzählt werden." - sofern sich der Leser nicht dazu ermutigen läßt, sich seine eigene Geschichte zu machen, was sicher die Intention des Autors ist.

\section{Auf der Suche nach dem wahren Willen: Traum und Wirklichkeit}

Alles, was Bastian in seinem Leben Ärger verursachte, seine Unsportlichkeit, seine dickliche Körperfigur usw., all das verändert sich ins Gegenteil, als er das Auryn, das Allmachtssymbol im Land Phantasien umgehängt bekommt. Das heißt: Phantasie kennt keine Grenzen, alles läßt sich erträumen - aber es ist eben nur Traum. Bisher hatte diese Flucht vor der eigenen Realität in eine Traumwelt der Geschichten den Bastian sich selbst eher verfremdet als daß es ihn zur Begegnung mit sich selbst, d. h. zur Selbstannahme, ermuntert hätte. Auf dem Auryn befindet sich die Inschrift: 'TU WAS DU WILLST' - aber für Bastian wird es ein langer Lernprozess, bis er diese Worte in ihrem wahren Sinn verstehen kann. So wird seine Reise und sein Erlebnis im Reich Phantasien zur Suche nach dem wahren Willen, wobei er mancherlei Irrwege gehen und schmerzliche Erfahrungen machen muß.

Mit dem Eintritt ins Reich der Phantasien merkt Bastian zuerst, daß er schön ist. Plötzlich verspürt er kein Bedürfnis mehr danach, bewundert zu werden. Er besteht Abhärtungsprüfungen und freut sich über die Tapferkeit, die ihm nun zu eigen ist. Diese Erfahrungen sind für ihn sehr grundlegend, denn erst dadurch, daß er sich selber mag, so wie er ist, kann er seine Wünsche 
kontrollieren und mit der Realität in fruchtbare Verbindung bringen. "Alles ist immer erst da, wenn ich es mir gewünscht habe. Und trotzdem ist alles viel großartiger und wirklicher, als ich mir's vorstellen könnte (S. 224)."

Diese Erfahrung bringt ihn schließlich zur Frage: "Wo kommen die Wünsche in uns eigentlich her?" (S. 227) Er spürt, daß diese Wünsche nicht nur nach Lust und Laune gebildet werden dürfen, denn jede Entscheidung fordert eine neue; er spürt nun, daß er ein Ziel haben muß, um richtig wählen zu können (vgl. S. 234). Um den mächtigen Retter vom Menschenland wieder sicher in seine Welt zurückbegleiten zun können, sollen die drei geeignetsten Helden durch Turniere ermittelt werden. Bastian besiegt Hykrion, den Mächtigsten unter den Starken, er besiegt Hysbald, den Gewandtesten unter den Flinken und auch Hydron, den Ausdauerndsten unter den Zähen. Als er am Ende auch noch gegen den Superhelden Hynreck siegt, weiß er, er braucht keine Beschützer mehr. Aber all dies war ihm gelungen, weil er noch das Machtsymbol Phanatsiens trug, und so erwacht in ihm der Wunsch, etwas zu tun ohne die Hilfe des Auryn, etwas, was nur er allein kann. "Ich will eine Geschichte erzählen, in der alle enthalten sind." (S. 258) Das ist sein erster Versuch, ohne fremde Hilfe wirklich selber etwas fertig zu bringen. Nach dem Besuch der Bibliothek der gesammelten Werke von B. B. B. spürt er, daß man Wünsche nicht nach Belieben hervorrufen noch unterdrücken kann. "Sie kommen aus tieferen Tiefen als alle Absichten." (S. 371) Der Held zu sein, war für ihn anfangs sehr überwältigend, aber je mehr er darüber reflektiert, umso mehr verspürt er Einsamkeit. Und so reift in ihm der Wunsch heranwenn auch als geringster - er möchte zu einer Gemeinschaft gehören (vgl. S. 372). Doch der Besuch in der Stadt Yskal im Nebelmeer sollte ihm noch eine weitere wichtige Erkenntnis bringen: Die Leute, die sich die Gemeinsamen nennen, sagen nur 'wir' nie 'ich' und scheinen zuerst eine ideale Gemeinschaft zu sein. Das Schiff, mit dem diese Leute auf das Nebelmeer hinausfahren, wird von der Vorstellungskraft der Schiffer angetrieben. Je mehr sie sich das gleiche vorstellen, umso größer ist die Kraft des Schiffes. Aber da sie sich nicht von einander unterschieden, war keiner unersetzlich - ihr Leben ist wie ihr Lied 'monoton', und Bastian versteht nun: es gab zwar Harmonie, aber keine Liebe. Er lernt sich hier mit dem Problem der Anpassung an eine Gruppe auseinanderzusetzen, wobei er merkt, daß es wichtig ist, seine Eigenständigkeit zu bewahren (vgl. S. 377). Er will nun plötzlich nicht mehr der Größte, Stärkste oder Klügste sein - er möchte nur noch geliebt werden. Damit hat er auch endgültig den verführerischen Einfluß der Xayide, der sehenden Hand, überwunden, die sich in seine Gunst eingeschlichen hatte. Diese Xayide hatte mit ihren Schmeicheleien Atreju verleumdet und so die Freundschaft in Gefahr gebracht. Auf ihre Worte hin wurde der Held 
Bastian so stolz, daß er sich selber zum Herrscher über Phantasien hatte krönen lassen wollen. Doch der Tag der Krönung wurde zur großen Schlacht, in der Atreju zum eigentlichen Retter Phantasiens wird, da er das Unheil verhindern kann. Bastian gehen die Augen auf: Atreju war nicht sein Feind, sondern sein Retter. Jetzt kann er auch den Worten Atrejus Glauben schenken, daß er das Auryn ablegen und wieder in seine Welt zurückkehren müsse. Doch je mehr er zum eigenwilligen "Retter" Phantasiens geworden war, umso mehr hatte er auch die Erinnerung an seine Welt eingebüßt. Einerseits war dies zunächst ein notwendiger Prozess, da er seine bisherige Angst und Unsicherheit vergessen mußte. Er mußte seinen Minderwertigkeitskomplex vergessen, um zu sich selber zu finden in einem Neubeginn - gereift durch seine Erfahrungen in Phantasien. Seine diesbezüglichen Schuldgefühle kann ihm die Dame Aiuola im Änderhaus ausreden; "Der Weg der Wünsche ist nie gerade. Du hast zwar einen Umweg gemacht - aber es war DEIN Weg (S. 392)." Auch wenn Erinnerungen erlöschen, "nichts geht verloren - alles verwandelt sich" (S. 393). Und so wartet er bei der so mütterlich um ihn sorgenden Aiuola, bis sich sein letzter Wunsch herausgebildet hat: Er muß das Wasser des Lebens finden. Damit verbindet sich nun sein inniger Wunsch, selbst lieben zu können. In ferner Zukunft soll eine Zeit kommen, wo die Menschen die Liebe auch nach Phantasien bringen werden. Dann werden beide Welten nur noch eine sein (vgl. S. 394). So mußte er selbst Vater und Mutter vergessen, bis er nichts mehr hatte als seinen Namen und er dann "eingerollt wie ein ungeborenes Kind im Leib seiner Mutter lag in den dunklen Tiefen der Grundfesten Phantasiens und schlürte nach einem vergessenen Traum." Dabei reifte eine weitere Erkenntnis in ihm: Für das Wasser des Lebens mußte er auch das letzte vergessen, was er noch hatte: sich selbst, denn nur so wird er wirklich lieben können. Fest entschlossen, dem Vater das Wasser des Lebens zu bringen, legt er das Auryn vor Antreju nieder. Dieses löst sich auf und wird so zum Tor zurück in seine Welt. Je näher sie dem Wasser kommen, kehrt Bastians ursprüngliche Gestalt zurück, und als er trinkt, verspürt er "Freude, er selbst zu sein" (S. 416). Er war neugeboren.

Aber noch etwas hatte Bastian beim Abschied von Antreju gelernt. Als ihm von der weißen Schlange zur Bedingung gemacht wird, daß er alle Geschichten zu Ende bringe, stammelt Bastian: "Alle Geschichten? Dann kann ich nie mehr zurück. Dann war alles umsonst." (S. 418) Es gebe nur einen Ausweg, wenn er jemand finde, der das für ihn übernehme. Als Bastian nahe der Verzweiflung ist, springt Antreju für ihn ein: er werde dies übernehmen. Voll Dankbarkeit stammelt Bastian: "Atreju, Atreju! Das werd' ich dir nie vergessen!" Atreju lächelt: "Gut, Bastian, dann vergißt du auch Phantasien nicht." (S. 418) Diese teure Geschenk einer wahren 
Freundschaft, die beide Welten unvergeßlich verbindet, wird so zum Retter für Bastian.

Mit dem Selbstvertrauen, das ihm seine Reise nach Phantasien geschenkt hat, klettert er das Baugerüst hinunter. Zwar gelingt ihm dies nicht in traumhafter Wendigkeit, aber er ist ein normaler Junge, mit Mut, Ausdauer und Zähigkeit. Dadurch aber, daß er sich geändert hat, ändert sich auch die Einstellung der Umwelt zu ihm: Der Vater umarmt ihn voll Freude (S. 422): "Von jetzt an", sagte der Vater mit einer ganz veränderten Stimme, "von jetzt an wird alles anders werden mit uns, meinst du nicht?"

Letztlich war es Bastian selbst, der sich dieses Phantasien ausgedacht hat, der dort die Begegnungen ersonnen hat und zu den verschiedenen Einsichten gekommen ist: er selbst hat zu seinem wahren 'ich' gefunden. Dazu mußte er alle seine Wünsche, die aus seinem Innern aufkamen, akzeptieren, die bewußten wie unbewußten. Er mußte lernen, seine Wünsche zu kontrollieren und für sein Ziel nützlich zu machen. Nur so bewahrheitet sich der Satz des Auryn "TU WAS DU WILLST" - denn das Problem besteht darin, das Wahre, das Richtige zu wollen. Solange Bastian davon träumt, ein großer Sportler zu sein, wird er nie zufrieden und glücklich sein können. Um aber das Gerüst von der Dachkammer hinunterzusteigen, dazu reicht jetzt seine Kraft und Beweglichkeit, und er kann mit sich zufrieden sein. Er hat gelernt, seine Wünsche anzupassen, ohne dabei seine Träume als Träume. aufzugeben. Träume dürfen nicht in Flucht vor der Realität ausarten, sondern nur in der gegenseitigen Befruchtung von Traum und Realität kann man zu wahrer Liebe finden, und dazu gehört auch, daß man vergessen kann.

\section{Abschließendes}

Der Autor M. Ende, der besonders in seiner Zeit als Schauspieler ein Verehrer von B. Brecht war, störte sich aber immer mehr an der zu politischen Literatur, wie er in einem Interview im NHK am 26. Juli 1986 in Verbindung mit dem 20. Weltkongress für Kinderbücher bekannte. Er sagte dort, daß die Sonnenblume von v. Gogh mehr besage als all diese Bücher von Grass, Brecht und Böll, die mit ihrer gesellschaftskritischen Literatur die Welt zu bessern versuchen, und das Buch "Die Unendliche Geschichte" sei eine Antwort auf diese Bücher. Dabei ging es Ende nicht nur um eine Wiederentdeckung der Phantasie für eine $\mathrm{zu}$ realistisch denkende Gesellschaft. Er wollte mit den so zahlreichen Traumwesen aus verschiedensten Kulturen auch nachdenklich stimmen über unsere Wertmaßstäbe wie industrialisiert $=$ kultiviert bzw. unterentwickelt $=$ Kulturmangel. So ein Denken sei zu materiell, man vergesse den 
Symbolreichtum in Kunst, Musik und Lebensgewohnheiten bei diesen Völkern. Mit diesem Anliegen verbindet der Autor seine sozialpolitische Kritik an der Gegenwartsliteratur, die aber nie direkt ausgesprochen wird, da dies eben gerade gegen sein Prinzip des "guten Buches' sprechen würde. Sehr interessant ist auch die rein optische Trennung der "Unendlichen Geschichte", die in grünen Lettern gedruckt ist, und der Geschichte des Lesers Bastian, die in roten Buchstaben ihren Verlauf schildert. Gerade durch diese Trennung in eine Geschichte als Lesestoff und in eine Erzählung über die Vorgänge beim Leser sprengt diese Phantasieerzählung den Rahmen eines Kunstmärchens, wie auch den eines Märchenromans. Die Schwierigkeit der Zuordnung zu einer bestimmten literarischen Gattung wird von vielen Kritikern als negativ beurteilt, doch entspricht dieses Moment sicher auch einem wichtigen Anliegen des Autors, da der Lesestoff erst in der Phantasie, im Engagement des Lesers zur eigentlichen Geschichte wird.

Die große Zahl an "drop outs" und bedauerliche Statistiken von Kinderund Jugendselbstmorden unterstreichen die Wichtigkeit in der Erziehung, Kindern zu helfen, einen Sinn im Leben zu finden. Dazu sind viele Wachstumserfahrungen nötig. Das Kind muß in seiner Entwicklung lernen, sich selbst immer besser zu verstehen. Nur dann vermag es auch, andere zu verstehen und dadurch eine befriedigende und sinnvolle Beziehung mit ihnen herzustellen. Um einen tieferen Sinn zu finden, muß man fähig werden, die engen Grenzen einer egozentrischen Existenz zu überschreiten und darauf zu vertrauen, daß man einen bedeutsamen Beitrag zum Leben leisten wird - wenn auch nicht gleich, so doch in der Zukunft. Diese Überzeugung ist notwendig, wenn jemand mit sich selbst und mit dem, was er tut, zufrieden sein soll.

Um den Wechselfällen des Lebens nicht hilflos ausgeliefert zu sein, muß man seine inneren Kraftquellen erschließen, so daß Gefühle, Phantasie und Intellekt einander unterstützen und bereichern. Unsere positiven Gefühle verleihen uns die Kraft, unseren Verstand zu entwickeln.

Unter diesem Gesichtspunkt ist ein großer Teil der Literatur, die dazu bestimmt sein soll, Geist und Persönlichkeit des Kindes zu entwickeln, sicherlich sehr unzulänglich, da gerade jene Fähigkeiten, die es am meisten braucht, um mit seinen inneren Problemen fertig zu werden, nicht geweckt und angeregt werden. Viele Vorschulbücher und Fibeln zum Lesenlernen verfolgen den Zweck, eine notwendige Fertigkeit zu lehren, vernachlässigen dabei die Sinnfrage. Die meisten Kinderbücher wollen unterhalten und informieren, aber sie sind inhaltlich oft schal, weil sie kaum etwas vermitteln, was für das Kind von tieferer Bedeutung wäre. Die Aneignung von Fertigkeiten wie z. B. das Lesenlernen wird entwertet, wenn das, was dann gelesen wird, nichts enthält, was für das eigene Leben wichtig ist. 
Wir alle neigen dazu, die künftigen Vorteile einer Tätigkeit danach einzuschätzen, was sie uns heute bietet. Besonders gilt das für das Kind, das viel stärker als der Erwachsene in der Gegenwart lebt. Sicher hat das Kind nur eine ganz verschwommene Vorstellung davon, wie seine Zukunft sein wird und was sie erfordern könnte. Die Erwartung aber, das Lesenlernen werde später einmal das Leben bereichern, entpuppt sich allzu oft als leeres Versprechen, wenn die Geschichten, die dem Kind vorgelesen werden oder die es selbst liest, nichtssagend sind.

In seinem Buch 'Kinder brauchen Märchen' fordert Prof. Bruno Bettelheim: "Soll eine Geschichte ein Kind fesseln, so muß sie es unterhalten und seine Neugier wecken, um aber sein Leben zu bereichern, muß sie seine Phantasie anregen und ihm helfen, seine Verstandeskräfte zu entwickeln und seine Emotionen zu klären. Der Inhalt muß auf seine Ängste und Sehnsüchte abgestimmt sein, seine Schwierigkeiten aufgreifen und zugleich Lösungen für seine Probleme anbieten. Dabei dürfen die kindlichen Nöte nicht verniedlicht werden - diese müssen in ihrer Schwere ernst genommen sein, damit das Vertrauen des Kindes in sich selbst und seine Zukunft gestärkt wird." (3)

Die "Unendliche Geschichte" ist eine exakte Antwort auf diese Anforderungen und der Erfolg des Buches beweist, daß viele Kinder und Erwachsene darin echte Bereicherung gefunden haben.

\section{Literaturverzeichnis}

1. Nachrichtenmagazin "Der Stern", Nr. 34, 18. August 1983, Artikel 'Die Phantastischen kommen', S. 32

2. Michael Ende, "Die Unendliche Geschichte", K. Thienemann Verlag, Stuttgart, 1979, S. 139. N. B.: Alle weiteren Seitenangaben zum Buch beziehen sich auf diese Ausgabe.

3. Zitat aus der Rede von M. Ende auf dem 20. Weltkongress für Kinder- und Jugendbücher in Tokyo am 18. 8. 1986. Der Originaltext im Englischen lautet: "I am not guided by pedagogical or didactic principles while I work... I have given my books this visible form for poetic and artistic reasons alone".

4. Knaurs Lexikon der Symbole, Hrsg. Hans Biedermann, Droemer Knaur Verlag, München, 1989, S. 454-456

5. Bruno Bettelheim, "Kinder brauchen Märchen", dtV-Verlag, München, 1977, S. 11 
「はてしなき物語」の現代社会に対してのメッセージ

中尾 美恵子

産業医科大学医療技術短期大学非常勤講師（ドイツ語）

要「旨：「シュピーゲル」誌のベストセラーリストには，ミヒャエルエンデの作品「はてし なき物語」が 3 年 3 カ月連続 1 位を占め続けた。これはドイツ語の本で戦後最大の 成功作品といえる．「読者」の少年バスチャンがその本の中に自ら入って，そのファ ンタジーの世界の中で多くを経験し, 迷い, 問い, 考え, 失敗しつつ, 精神的成長 を遂げ，自己に目覚めるさまを，子供向けに，文章的には平易に，ファンタスチッ クな物語として書かれている，またそれと同時に，登場人物やイメージの多義性で もって，自由自在に物語の中で遊ぶという巧みな構成がなされ，ファンタジーを躍 動させる機会を失った 20 世紀の大人に間接的ではあっても, ファンタジーやポエ ジーが，大人の世界に広がれば，現実社会のゆがみも是正される可能性を示唆した 含蓄のある大人向けの作品でもある.

JUOEH（産業医大誌）17 ( 3 ): 215-228 (1995) 